\title{
The transient observation-based particle (TOP) model and its potential application in radiation effects evaluation
}

\author{
Sylvie Benck ${ }^{1, *}$, Mathias Cyamukungu ${ }^{1}$, Juan Cabrera ${ }^{1}$, Laura Mazzino ${ }^{3}$, and Viviane Pierrard ${ }^{1,2}$ \\ 1 Center for Space Radiations (CSR), Institute of Research in Mathematics and Physics, Université Catholique de Louvain, \\ B-1348 Louvain-la-Neuve, Belgium \\ *corresponding author: benck@spaceradiations.be \\ 2 Belgian Institute for Space Aeronomy (BISA), B-1180 Brussels, Belgium \\ 3 Department of Physics, University of Alberta, 11322-89 avenue, Edmonton, Alberta, Canada
}

Received 8 February 2011 / Accepted 21 December 2012

\begin{abstract}
The evaluation of the radiation hazards on components used in space environment is based on the knowledge of the radiation level encountered on orbit. The models that are widely used to assess the near-Earth environment for a given mission are empirical trapped radiation models derived from a compilation of spacecraft measurements. However, these models are static and hence are not suited for describing the short timescale variations of geomagnetic conditions. The transient observation-based particle (TOP)-model tends to break with this classical approach by introducing dynamic features based on the observation and characterization of transient particle flux events in addition to classical mapping of steady-state flux levels. In order to get a preliminary version of an operational model (actually only available for electrons at low Earth orbit, LEO), (i) the steady-state flux level, (ii) the flux enhancements probability distribution functions, and (iii) the flux decay-time constants (at given energy and positions in space) were determined, and an original dynamic model skeleton with these input parameters has been developed. The methodology is fully described and first flux predictions from the model are presented. In order to evaluate the net effects of radiation on a component, it is important to have an efficient tool that calculates the transfer of the outer radiation environment through the spacecraft material, toward the location of the component under investigation. Using the TOP-model space radiation fluxes and the transmitted radiation environment characteristics derived through GEANT4 calculations, a case study for electron flux/dose variations in a small silicon volume is performed. Potential cases are assessed where the dynamic of the spacecraft radiation environment may have an impact on the observed radiation effects.
\end{abstract}

Key words. 2774: Radiation Belts - 7984: Space radiation environment - 2722: Forecasting - 7934: Impacts on technological systems - 7959: Models

\section{Introduction}

At the present time the evaluation of radiation hazards on components in space is mainly based on static empirical models following defined standards (e.g., ECSS-E-ST-10-04C). However, these static models are not suited for describing the short timescale variations that need to be taken into account for some categories of radiation effects. In addition, as these models are based on averages taken over large timescales, they fade away the characteristics of physical processes that drive the dynamic of particle flux variations. Recently, space weather preoccupations have therefore encouraged the development of dynamic models, either physics based (Beutier \& Boscher 1995; Fung 1996; Glauert \& Horne 2005; Koller et al. 2007; Fok et al. 2008; Albert et al. 2009; Subbotin \& Shprits 2009) or empirical (O'Brien \& McPherron 2003; Ling et al. 2010; Turner et al. 2011). The physics-based models are mostly data-driven physical models that incorporate a radial diffusion code and where the measured data provide the boundary conditions. The above-cited empirical models involve various approaches to describe electron dynamics and focalize on the forecasting of relativistic electrons at geosynchronous orbit. The transient observation-based particle (TOP) model is an empirical model that is based on the observation and parameterization of transient particle fluxes. This approach is expected to facilitate the investigation of the physical processes involved in the flux variations in the magnetosphere. In a first step (presented in this paper), the development of the TOP-model includes (i) the study of storm occurrence probabilities, (ii) the determination of particle flux during geomagnetically quiet periods (steady state), (iii) the study of the response of radiation belt particle flux during storm times, and (iv) the analysis of the flux decrease after the end of the storm: the derived model will be probabilistic, that is based on the probability distribution functions (PDF) of the flux variation parameters. In a second step, the development of a physical model of the event-driven flux variations as a function of the geomagnetic indices and/or solar wind parameters is foreseen.

Section 2 describes the data analysis procedure which includes the determination of the main parameters that are used in the model. The simulation procedure is described in Section 3 and the results are presented in Section 4. The discussion chapter (Sect. 5) describes how the deduced fluxes can find their application in radiation effects evaluation. The conclusions are given in Section 6. 
Table 1. Lower and upper limits of the channels for the SAC-C/ICARE energy grid.

\begin{tabular}{llllllllll}
\hline \hline & ch1 & ch2 & ch3 & ch4 & ch5 & ch6 & ch7 & ch8 & ch9 \\
\hline$E_{\min }(\mathrm{MeV})$ & 0.19 & 0.23 & 0.29 & 0.33 & 0.35 & 0.45 & 0.53 & 0.59 & 0.64 \\
$E_{\max }(\mathrm{MeV})$ & 0.25 & 0.29 & 0.35 & 0.39 & 0.45 & 0.51 & 0.59 & 0.65 & 0.76 \\
& ch10 & ch11 & ch12 & ch13 & ch14 & ch15 & ch16 & ch17 & ch18 \\
\cline { 2 - 9 } & 0.76 & 1.08 & 1.24 & 1.28 & 1.72 & 2.19 & 2.67 & 3.15 & 3.63 \\
$E_{\min }(\mathrm{MeV})$ & 0.88 & 1.36 & 1.60 & 1.72 & 2.20 & 2.67 & 3.15 & 3.63 & 4.11 \\
$E_{\max }(\mathrm{MeV})$ & & & & & & & &
\end{tabular}

\section{Data analysis}

\subsection{The electron data sets}

In this study of the low Earth orbit (LEO) environment, we use the electron data obtained by the SAC-C/ICARE(Falguère etal.2002; Ecoffet et al. 2005; Bourdarie et al. 2006) and DEMETER/ IDP (Parrot 2006; Sauvaud et al. 2006) instruments, located in a sun-synchronous circular orbit at an altitude of about $700 \mathrm{~km}$.

The ICARE radiation detectors are made of fully depleted solid-state detectors used in single and/or coincident and/or anti-coincident mode. The ICARE omni-directional differential electron fluxes (retrieved from http://www-mip.onera.fr/rermm/ $\mathrm{SACC} /$ ) are given within the energy range 0.19-4.11 MeV. Table 1 gives the lower and upper limits of the 18 energy channels. The electron flux data used within this study were acquired during the orbit time period December 2000 to September 2006. This data set was used to generate the steady-state flux (Sect. 2.2.1), to study the storm-related flux variations (Sect. 2.2.4), and to deduce the flux decay times (Sect. 2.2.2).

The flux measurements of the IDP instrument are achieved by counting particles that deposit energy in the $70-2340 \mathrm{keV}$ range in a $1 \mathrm{~mm}$ thick silicon detector. This energy interval is divided into 256 channels in "BURST mode" and 128 channels in "SURVEY mode" (the energy bins are equally spaced). For convenience, we grouped the energy channels in order to consistently work with 27 channels independent of the operating mode. Each new channel covers an energy range of about $90 \mathrm{keV}$. Spectra are given with a time resolution of 1 or $4 \mathrm{~s}$ depending on the operational mode (Burst or Survey). Also, it must be mentioned that only the 14 first channels out of the 27 show efficiency to electrons high enough to allow electron detection, the following channels may be a mixture of electrons and protons and the last channels detect more or less exclusively protons. Within this study the electron energy channels used, range from $162 \mathrm{keV}$ to $1.15 \mathrm{MeV}$ (11 equally spaced channels of about $90 \mathrm{keV}$ ) and the covered time period runs from August 2004 to March 2006. The IDP data set was only used in the flux loss timescale study (Sect. 2.2.2).

Readers can find more details on the data and how they were processed and compared in Benck et al. (2010).

\subsection{Model development}

The observation of transient particle fluxes was found to be a convenient approach to study the dynamic of the space radiation environment. This idea was inspired by the work of McIlwain (1996) who observed already in 1966, that the flux variations may follow a given behavior that he partially parameterized. Figure 1 shows the general features of the flux evolution as a function of time. Three main parameters can be identified: (i) the steady state which represents the minimum

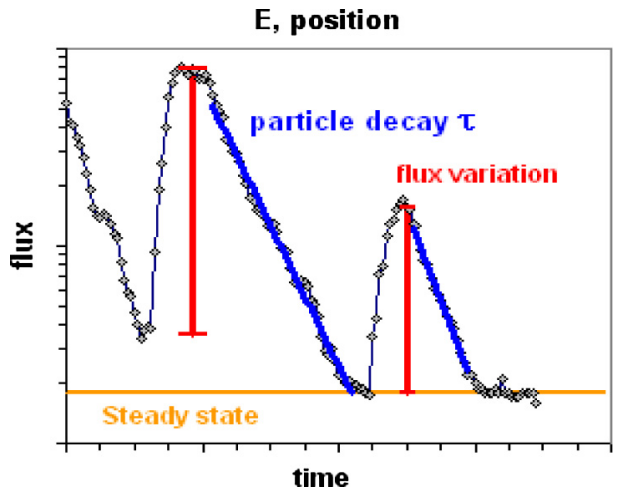

Fig. 1. Illustration of the general features of the electron flux behavior as a function of time for a given energy and position. The three main characteristics of the flux evolution are: (i) the steady state, (ii) the time constant $(\tau)$ of the flux decay, and (iii) the flux variation (enhancement or decrease) coupled to a geomagnetic storm event.

flux level to which the fluxes can progressively decay after flux enhancement (Sect. 2.2.1), (ii) the time constant $(\tau)$ of the flux decay following an event-driven flux increase (Sect. 2.2.2), and (iii) the flux variation (enhancement or decrease) coupled to a geomagnetic storm event (Sect. 2.2.4). An important parameter that cannot be observed in Figure 1 is the storm occurrence frequency. This parameter is obtained from Dst versus time analyses (Sect. 2.2.3).

The electron flux behavior shown in Figure 1 depends however on the energy of the electrons under investigation. In fact, it has been noticed that at energies above $3 \mathrm{MeV}$ the flux level is controlled by flux dropout during the initial phase of geomagnetic storms rather than by constant flux decrease characterized by a decay-time constant. Therefore, the present model development is limited to energies below $2 \mathrm{MeV}$.

\subsubsection{Determination of the steady state}

The steady-state fluxes are deduced from SAC-C data taken during a long period of low geomagnetic activity (from 1st May to 17 July 2004). For a given position ( $L, B$ coordinates) and energy, the flux measurements are simply averaged and recorded. The energy bins are as defined in Table 2. To obtain the fluxes for each energy bin an interpolation between detector channels is used. $L, B$ coordinates were calculated with UNILIB v2.20 library (http://trend.aeronomie.be/NEEDLE/unilib.html). The field strength $B$ is calculated using internal and external field models. The internal magnetic field component is calculated using the IGRF2000 coefficients extrapolated to the date of measurement. To calculate the external field component, the Tsyganenko 1989 model was selected with $\mathrm{Kp}$ set to $0^{+}$. The McIlwain $L$ parameter was determined using a magnetic dipole moment of $M=0.311653 \mathrm{GRe}^{3}$. 
S. Benck et al.: The transient observation-based particle model and its potential application

Table 2. Lower and upper limits for the model energy grid. The model presented here does not however consider energies above $1.5 \mathrm{MeV}$.

\begin{tabular}{|c|c|c|c|c|c|c|c|c|c|c|c|}
\hline & ch1 & $\operatorname{ch} 2$ & $\operatorname{ch} 3$ & $\operatorname{ch} 4$ & $\operatorname{ch} 5$ & $\operatorname{ch} 6$ & $\operatorname{ch} 7$ & $\operatorname{ch} 8$ & $\operatorname{ch} 9$ & $\operatorname{ch} 10$ & $\operatorname{ch} 11$ \\
\hline$E_{\min }(\mathrm{MeV})$ & 0.2 & 0.3 & 0.4 & 0.5 & 0.7 & 1.0 & 1.5 & 2.0 & 2.5 & 3.0 & 3.5 \\
\hline$E_{\max }(\mathrm{MeV})$ & 0.3 & 0.4 & 0.5 & 0.7 & 1.0 & 1.5 & 2.0 & 2.5 & 3.0 & 3.5 & 4.0 \\
\hline
\end{tabular}

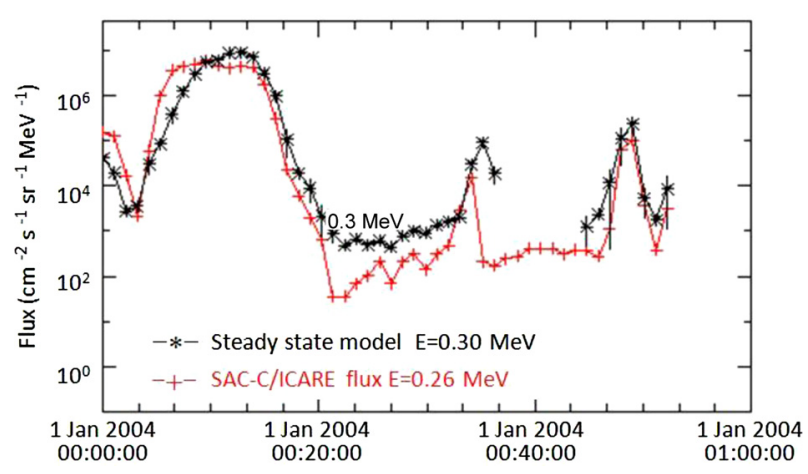

Fig. 2. Comparison between the results from the steady-state model (black) and flux measurements acquired by the SAC-C/ICARE instrument (red) along one half orbit starting from the southern polar region, toward the northern auroral region, through the SAA, on 1st January 2004

The steady state provides a lower limit of particle fluxes at a given position and it is recommended that such fluxes be used for future instrument cross-calibration in the same low activity geomagnetic conditions, since their variances are relatively low. Figure 2 shows a qualitative comparison between real-time measured fluxes and steady-state fluxes from the model. The measured fluxes were taken during a time period of low solar activity other than the one used to generate the model. It can be noticed that there is a good agreement between measured data and modeled fluxes.

Steady-state fluxes at a given position in space are assumed to be very stable and likely to be only affected by solar cycle and secular variation of the geomagnetic field. At LEO this dependence has been observed in the center of the inner belt $(1.4<L<1.8)$, where during the declining phase, the steady state increases to an out-of-scale maximum (saturation in the SAC-C/ICARE detector).

\subsubsection{Determination of the loss timescales}

The determination of the loss timescales from SAC-C and DEMETER data has been presented in a previous paper (Benck et al. 2010). More details on analysis procedure and results can be found therein.

The decay time (or loss timescale) is used in the model to simulate the flux variation after a storm (Fig. 1). In general, the steady-state flux level is reached after a determined lifetime which only depends on particle energy, position in space, and pitch angle. As the fluxes are assumed to decay exponentially to the quiet time level (steady state), the decay timescales, $\tau$, were obtained by fitting a linear function to the natural logarithm of the after-storm fluxes (Benck et al. 2010). Figure 3, taken from Benck et al. 2010, shows the mean decay timescales obtained for the indicated energy bins as a function of $\mathrm{L}$ for the region $1.6 \leq L<5$ and a common region $0.22<B<0.46 \mathrm{G}$. Cases are observed in which enhanced fluxes do not decay according to the characteristic lifetimes. Investigation of such behaviors reveals itself to be a powerful tool for the

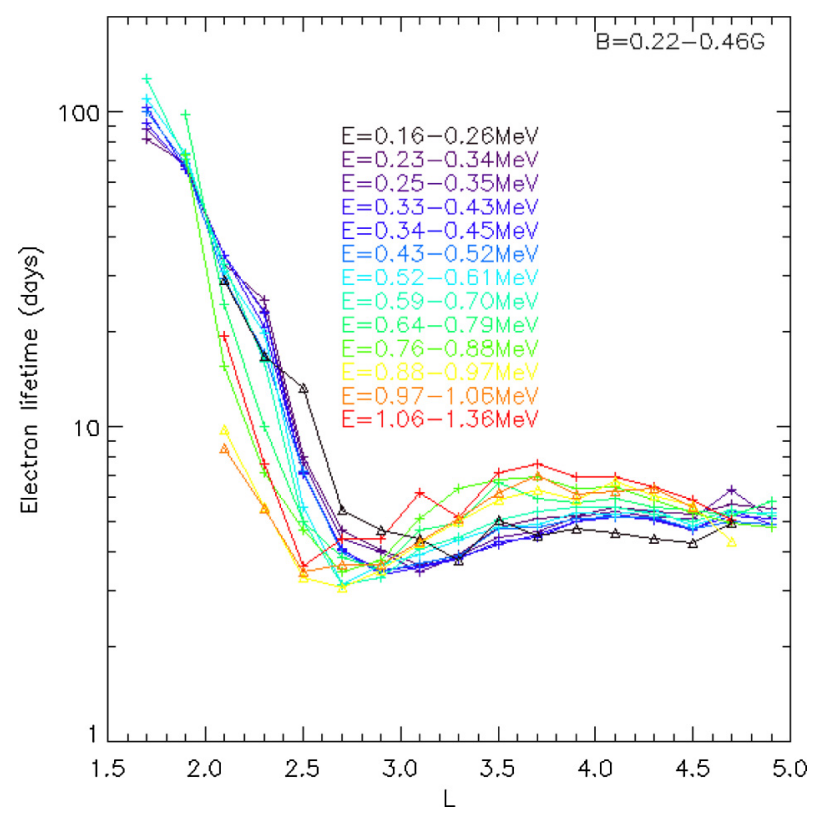

Fig. 3. Measured mean electron loss timescales versus $L$ for the indicated energy ranges (Benck et al. 2010).

identification of the mechanisms that affect space particle flux variations, but is outside the scope of this paper.

\subsubsection{Analysis of the storm occurrence frequency}

Fifty years of geomagnetic storm (GS) events characterized by their minimum Dst have been analyzed. The data set (Dst as a function of time with a time resolution of $1 \mathrm{~h}$ ) was downloaded from the World Data Center for Geomagnetism (WDC) and consists of hourly values of Dst from 1957 to 2007. A Fast Fourier Transform with a low-pass filter (fifth-order Butterworth filter) was applied to the data set. The maxima within the filtered data were detected and their time of occurrence was recorded. Subsequently, within the time interval determined by two consecutive detected maxima, the lowest value (minimum) in the Dst original data set was searched for in order to determine the exact time of Dst minimum and the magnitude of the corresponding storm. If multiple events occurred within a small time interval ( $\leq 2$ days), the filter automatically merged them into one single event, and only the lowest Dst value was taken. GS were detected when their Dst values were less than $-50 \mathrm{nT}$. All substorms, defined as magnetic storms with $D s t$ values greater than $-50 \mathrm{nT}$, were not considered in the analysis. Additionally, to each of the 1224 identified storm events, a storm "begin-" and "end-" time was associated, defining a time interval within which the storm-time flux variation was analyzed (Sect. 2.2.4).

Two probability distributions were used to characterize storm-time events: the geomagnetic storm magnitude $(D s t)$ distribution and the waiting time distribution (the waiting time being defined as the time interval between two consecutive storms). For convenience the " $-D s t$ " data is used in the figures hereafter. 

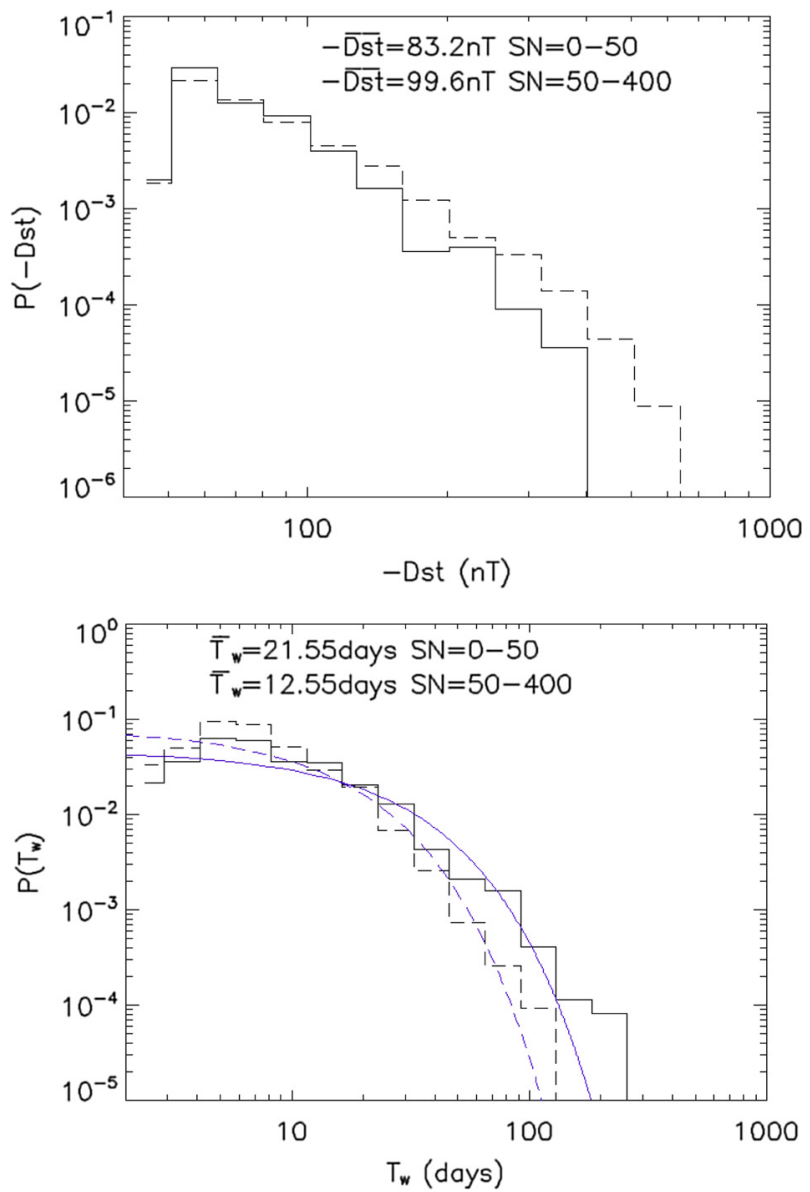

Fig. 4. Dst probability distributions (upper panel) and waiting time distributions (lower panel). The continuous lined histograms correspond to the quiet solar activity periods and the dashed lined histograms correspond to the high solar activity periods. The blue continuous and dashed lines on the lower panel represent a fit of the probability functions with a poissonian distribution: $P\left(T_{\mathrm{w}}\right)=$ $r \exp \left(-r T_{\mathrm{w}}\right)$ with $r=1 / \overline{T_{\mathrm{w}}}$

To get a preview of the Dst and waiting time $\left(T_{\mathrm{w}}\right)$ distributions, the data set was subdivided in two solar activity periods: The quiet (active) solar periods are defined as the periods when the smoothed sunspot number is below (above) 50. Figure 4 shows the Dst and waiting time distributions for the active and quiet periods. It can be observed that during the - quiet solar periods, the probability of having a high intensity storm is lower than during the active solar periods, and that the waiting times between storms are generally much longer. The storm occurrence is close to a poissonian process when amplitude is not taken into account. A fit has been done through the waiting time distributions giving a mean waiting time $\left(\overline{T_{\mathrm{W}}}\right)$ of 21.5 and 12.5 days for the quiet and active solar phases, respectively.

\subsubsection{Determination of the flux enhancements}

The SAC-C data covers the period from December 2000 to September 2006. During this period of time 138 geomagnetic storms with $|-D s t|>50$ nT were identified (Sect. 2.2.3). However, the SAC-C data acquisition was sometimes interrupted and no flux data was given during six hours, leaving holes in the fluxtime evolution. These periods needed to be discarded from the analysis. Also, the fluxes measured during the declining phase of solar cycle 23, between November 2002 and January 2004, were discarded from the analysis due to unstructured flux
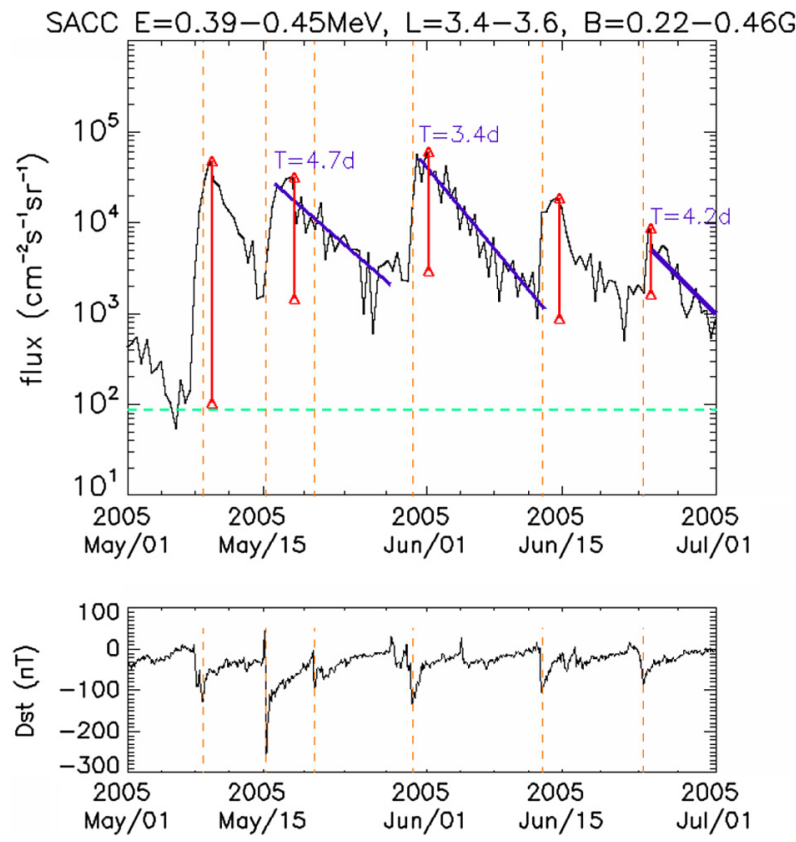

Fig. 5. The upper panel shows SAC-C electron fluxes as a function of time for the indicated energy range and position. The green dashed line corresponds to the steady state at that position. The orange dashed vertical lines indicate the time of occurrence of a storm event, corresponding to a Dst minimum (lower panel). The red vertical segments correspond to the observed flux enhancements. The blue lines show the result of the fitting procedure of the decreasing after-storm fluxes with an exponential function. The resulting lifetimes are also indicated (Benck et al. 2010).

behavior (i.e., no general features like illustrated in Figure 1 could be observed), leading to increased average flux level. The analysis of this specific period is outside the scope of this paper. Therefore, results could only be obtained for about 87 storms (depending on the energy and position). Figure 5 shows an example for the determination of flux variation (deltaF) from the SAC-C/ICARE electron flux data as a function of time.

In order to get a first version of an operational engineering model, the flux enhancements distribution functions were established and the first dynamic model skeleton with these as input parameters was issued in the course of 2010. The model will subsequently be fed with new data as they will become available and it will be refined using inputs from a physical parameter analysis. Figure 6 shows the electron flux variation probability distribution function at LEO for $L=3.4,4.8$ $(B=0.22-0.46 \mathrm{G})$ and $E=0.29-0.35,0.64-0.76 \mathrm{MeV}$. For these energy bins and positions most of the storms induce flux increase.

Additionally, for each determined flux variation event, the time between minimum Dst and after-storm maximum flux level is registered and its corresponding "time interval" probability distribution function is derived (Fig. 7). For each position and energy, the PDF of the "time interval" defines a time factor used in the dynamic model simulation. Although the Dst time evolution is used to characterize the time sequence of the storm development, no obvious relationship was observed between the strength of the storm (determined by the absolute value of Dst minimum) and the induced flux variations. However, a variation of the type of storm, such as Coronal Mass Ejections (CMEs), Corotating Interaction Regions (CIRs) or multiple storm that includes both types, was reported to result in a 
S. Benck et al.: The transient observation-based particle model and its potential application
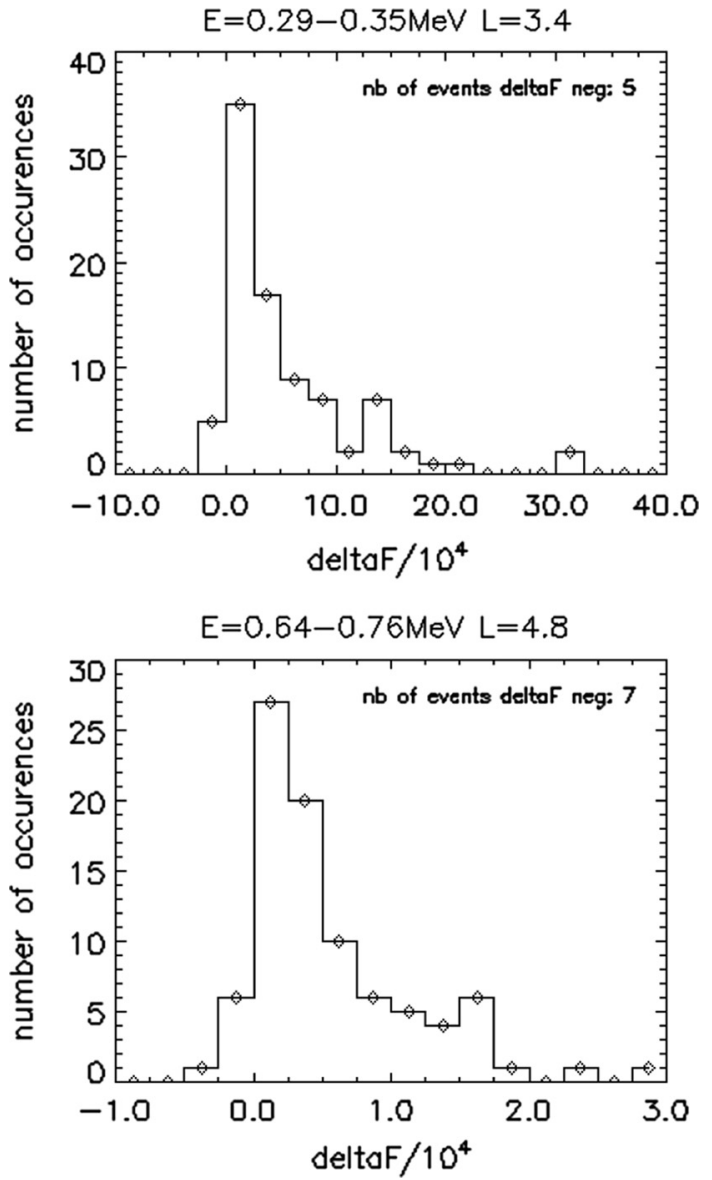

Fig. 6. Flux enhancement (deltaF in $\mathrm{cm}^{-2} \mathrm{~s}^{-1} \mathrm{sr}^{-1}$ ) distribution functions for the indicated energies and positions as deduced from the SAC-C data.

difference on the recovery time interval, and therefore, the time between Dst minimum and maximum flux, as well as the induced particle flux response (Kataoka \& Miyoshi 2006).

\section{The simulation procedure}

\subsection{The storm-time event generation}

Based on the evaluated characteristics of the storm-time fluxes, it is possible to simulate the flux dynamics for a given mission as a function of orbit position and geomagnetic activity. When using classical static flux models, the flux encountered at a given positions is always the same. This is not the case when using the dynamic model.

For the model application, 5 months of grid positions (700 km altitude sun-synchronous orbit) were calculated by the SPENVIS orbit generator (http://www.spenvis.oma.be), recorded in a CVS file, and used as input orbit data for the model. Then for each geographic position given in the CVS orbit file, the following coordinates are determined using the UNILIB v2.20 library: magnetic field strength $B$, Mcllwain parameter $L$, magnetic latitude, invariant radius.

For each position, the corresponding steady-state fluxes are established. The energy bins are homogenized so that the resulting fluxes are given for an energy grid as shown in Table 2 (Sect. 2.2.1). This is necessary in case data from many instruments with different energy bin definitions are used (see Sect. 2.1).

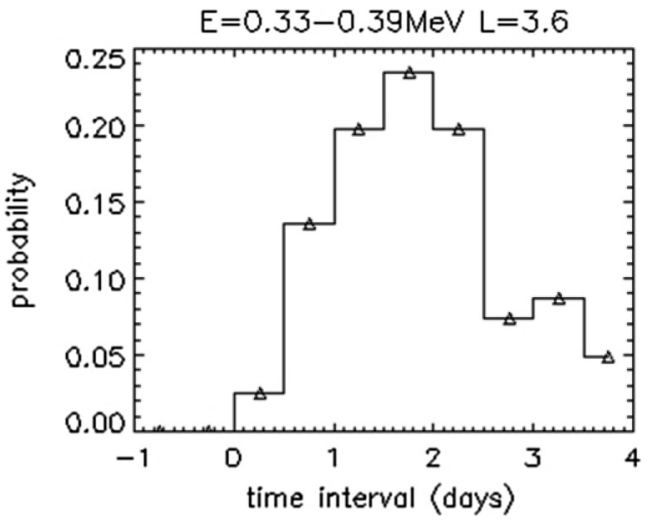

Fig. 7. Probability distribution function for the time interval (time measured between the instant of Dst minimum and instant of flux maximum).

Then for each position in the CVS file the dynamic flux parameters (PDF of the flux variation, PDF of the time interval, decay-time constants, and corresponding uncertainties) are calculated (interpolated) to comply with the above-mentioned homogenized energy binning.

Finally within the time span extracted from the input CVS files, the storm occurrence times are generated, depending on which phase within the solar cycle is to be simulated. The storm times are generated assuming that the waiting time distributions are poissonian with a mean waiting time $\overline{T_{\mathrm{W}}}$ between storms of 21.5 days for minimum and rising phase of the solar cycle and 12.5 days for maximum and declining phase of the solar cycle. However, to avoid a superposition of two storm events, it is imposed in the storm-time generation procedure that the time between two storms must be higher than 1.35 days which corresponds to the average time observed between the onset of a storm and the reaching of minimum Dst. This has as a consequence that the generated mean storm waiting time $\overline{T_{\mathrm{W}}}$ is somewhat larger than the original one.

\subsection{The flux variation generation}

For each storm generated during the 5-month interval, a random number (random_storm_strength_index) between 0 and 1 is generated, that determines the flux enhancement strength. A higher number corresponds to a higher flux enhancement. Here it is assumed that the flux variations are correlated for all energies and positions. It was observed that this is usually true when considering the flux variation for different energies at a fixed position, but this is only partly true when comparing the flux enhancements for a given energy at different positions. From the PDF of the flux enhancement parameter, the corresponding cumulative distribution functions (CDF) are deduced. Reporting the generated random number on the CDF abscissa, the corresponding flux enhancement for that storm can be deduced.

\subsection{The decay-time generation}

Similarly as for the flux variations, for each simulated storm, a random number between 0 and 1 is generated, that will determine the flux decay-time constant. For a given storm, this number is the same for all energies and positions. The probability distribution is assumed to be quasi-normal shaped with a mean value corresponding to the mapped average decay time $\tau$ and a variance $\sigma_{\tau}$ (given by the uncertainty). However, to avoid 
extreme, not observed values, samples exceeding $\tau \pm 1.5 \sigma_{\tau}$ were excluded.

\subsection{The simulation result}

For each position and energy, the flux evaluation depends on what happened in the past. Therefore, a procedure must be elaborated that calculates the flux at the given time and position, for each energy, by taking into account all the storm events that have happened before and their time sequence. The fluxes are calculated considering some rules:

(a) The flux evolution during a storm event is divided into three time intervals:

[Time of storm occurrence - Time of Dst minimum]:

Between the onset of the storm and the reaching of minimum Dst some time elapses (on average 1.35 days) within which the flux evolves as if nothing has happened, but the occurrence of a new storm is not allowed (see Sect. 3.1). This is the time period during which electron dropouts occur and the flux evolution in this time interval has not been parameterized yet.

[Time of Dst minimum - Time of maximum flux]:

Within this time interval the flux increases linearly of an amount corresponding to the defined flux variation for that event. To define this time span, the mean value is taken for each energy and position from the PDF of the time interval defined in Sect. 2.2.4.

[Time of maximum flux - Time of next storm occurrence]:

After reaching the maximum, the flux decreases exponentially according to the determined decay-time constant, until reaching the steady state (if no new storm occurs). In some cases the flux variation may be negative (see Fig. 6); in that case if at the time of flux "maximum", the flux level is above the steady state, the flux decreases exponentially, otherwise it is increased exponentially according to the same decay-time constant. (Such cases have not yet been analyzed and some approximations have to be done to take them into account.)

(b) Once a new storm occurs, the effects of the preceding storm are annihilated when the time of $D s t_{\min }$ is reached for this new storm, and only the characteristics of the new storm are taken into account in the evolution of the flux level.

\section{Results}

Figure 8 shows the flux as a function of time within a given position bin $(L=4.0-4.2, B=0.38-0.40 \mathrm{G})$ for the central energy $0.60 \mathrm{MeV}$. In the example shown, to each storm event, corresponds a flux enhancement, the size of which is determined by the intensity of the storm (cf., height of the vertical red line). The blue line corresponds to the mean flux at that position. From this graph it can be observed that most of the time the instant fluxes diverge strongly from the mean value.

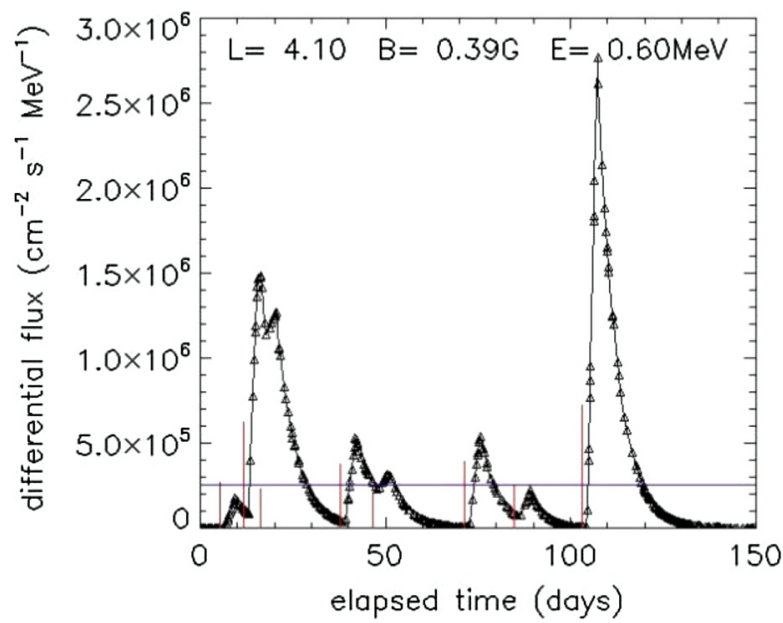

Fig. 8. Simulated flux as a function of time for the indicated energy and position. The red vertical lines indicate the times of occurrence of the storms. The storm waiting time was fixed to 12.5 days. The blue line represents the mean flux in that position bin for the given period of simulation.

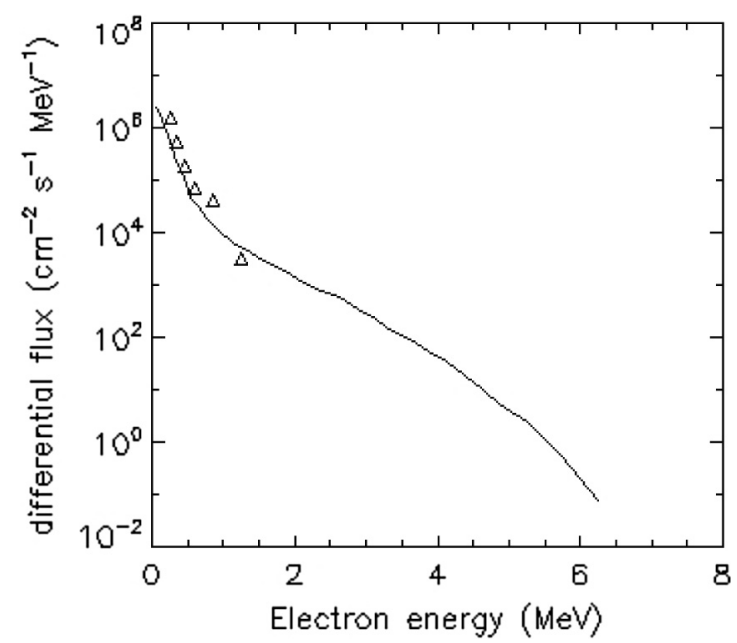

Fig. 9. Average differential flux on a sun-synchronous LEO orbit of $700 \mathrm{~km}$ altitude as obtained with the TOP-model (triangles). The solid curve is the flux calculated by the AE8-MAX model.

From the instant fluxes along the orbit, the average differential flux may be deduced and compared to calculations from the static AE8-MAX model (Vette 1991). Figure 9 shows the average differential fluxes from the TOP-model (triangles) and from the AE8-MAX model (continuous lines). Typically, the TOPmodel average differential fluxes are about two times higher than the fluxes from the AE8-MAX model. By analyzing the flux prediction per region of interest, it was found that although some overestimation (depending on the energy) comes from the upper $L$ region (see Fig. 10), most of the difference is however observed in the inner belt region $(L<2.5, B>0.22 \mathrm{G})$ outside the South Atlantic Anomaly (SAA). The problem here is twofold: First, the storm occurring on day 103 is very strong (random storm strength index $=0.96$ ) and therefore predicts a very high flux enhancement in all regions. Moreover, the inner belt regions $(L<2.5)$ have a very large flux decay-time constant rising up to about 100 days, and so this flux does not have time to decrease within the remaining time of the simulation period. This shows that the simulation time for the 
S. Benck et al:: The transient observation-based particle model and its potential application

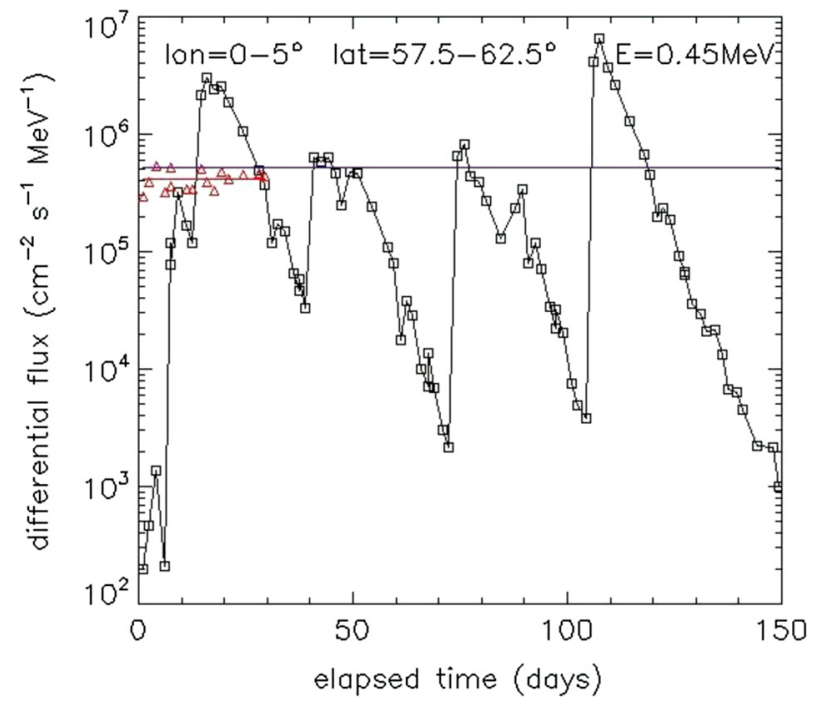

Fig. 10. Differential flux calculated for the defined position bin for the indicated energy during the considered simulation time period: the black squares and curve are the result from the TOP-model. The blue horizontal line gives the average differential flux for $0.45 \mathrm{MeV}$ encountered in that position bin based on the dynamic model fluxes. The red triangles are the result from the AE8 model (which was only run for a period of 30 days). The red horizontal line gives the corresponding average differential flux.

dynamic model should be extended in order to average over a longer time period. Secondly, the flux enhancement distribution functions are established with a bin in deltaF (see Fig. 6) that is based on the value of the highest flux enhancement ever encountered. As a consequence, the resolution is not sufficient on the low deltaF side. The resolution will be progressively improved as new data become available. The first version of the TOP-model does not include any dynamic for the SAA (defined as region where $B<0.22 \mathrm{G}$ ). In fact, it must be mentioned that in the center of the SAA $(B<0.18 \mathrm{G})$ the SAC-C data tend to saturate, therefore giving only a lower limit for the flux levels in that region. Non-saturated data set will help to improve the description of the radiation flux dynamics for this region.

Figure 10 shows, for a given position bin (latitude $=0^{\circ}-5^{\circ}$, longitude $\left.=57.5^{\circ}-62.5^{\circ}\right)$ and energy $(0.45 \mathrm{MeV})$, the evolution of the differential flux during the considered simulation time period: The black squares and curve are the results from the TOP-model. The dynamic feature of the flux is well illustrated: the steady state gives a lower flux limit of about $10^{3}$ electrons $/\left(\mathrm{cm}^{2} \mathrm{~s} \mathrm{MeV}\right)$ for $E=0.45 \mathrm{MeV}$ at that position, while the dynamics of the radiation belts can raise this flux up by four orders of magnitude. The blue horizontal line gives the corresponding average differential flux for $0.45 \mathrm{MeV}$ encountered in that position bin. The red triangles are the result from the AE8 model (which was only run for a period of 30 days). The time variation in the AE8 model comes from the fact that the defined position bin has a given extension and the orbit trajectory within the bin is not the same at each crossing. The red horizontal line gives its corresponding average differential flux. The steady state is far below the average differential flux levels predicted by AE8-MAX and TOPmodels. Obviously the dynamics of the radiation belt raise the average differential flux to its actual value. The storm waiting time (12.5 days were taken, see Sect. 2.2.3) is the factor that determines the number of occurrences of flux variations and hence is partly responsible for the final flux level.

The model calculations may be compared to observations in-sample (out-of-sample comparison for solar cycle maximum conditions is not possible in this case due to unavailability of data). The comparisons concentrate on the outer radiation belt as this regions shows a higher dynamic compared to the more stable inner belt. Figure 11 shows the time evolution of the 0.5 day averaged fluxes (in $\mathrm{cm}^{-2} \mathrm{~s}^{-1} \mathrm{sr}^{-1} \mathrm{MeV}^{-1}$ ) as deduced from SAC-C data for a period of 150 days during solar maximum (blue continuous lines) for two position bins $L=3.3-3.4, B=0.22-0.46 \mathrm{G}$ (upper panel) and $L=4.8-5.0$, $B=0.22-0.46 \mathrm{G}$ (lower panel). The discontinuous horizontal blue lines give the related fluxes averaged over the whole 150 day time period. The model results for 150 days (green lines) are superposed to the selected time period. It cannot be expected from the model to follow exactly the measured data, as in the simulation, the storms were generated randomly. The aspects that should be considered are the general features, that is the flux range, the number of flux enhancements, and the average resulting flux for a large time period. In the upper panel for $L=3.2-3.4$, it can be observed that the number of measured flux enhancements is 8 while the number of clearly observed flux enhancements generated by the model is 7 . In the lower panel, for $L=4.8-5.0$, it is difficult from the SAC-C data to determine a precise number of flux enhancements. However, it can be observed that in both panels the range of the flux variations is well reproduced by the model. In the upper panel it can also be seen that the flux decay is reproduced by the model. The mean flux values in $\mathrm{cm}^{-2} \mathrm{~s}^{-1} \mathrm{sr}^{-1} \mathrm{MeV}^{-1}$ when the fluxes are averaged over the whole period of 150 days are as follows: For $L=3.2-3.4: 72620$ for SAC-C data $(E=0.33-0.39 \mathrm{MeV})$, 83720 for the TOP-model $(E=0.3-0.4 \mathrm{MeV})$, and 29782 for the AE8-MAX model $(E=0.3-0.4 \mathrm{MeV})$. For $L=4.8-$ 5.0: 24446 for SAC-C data $(E=0.45-0.51 \mathrm{MeV}), 29464$ for the TOP-model $(E=0.4-0.5 \mathrm{MeV})$, and 29969 for the AE8-MAX model $(E=0.4-0.5 \mathrm{MeV})$. The 150 days averaged flux values from the TOP-model vary up to $\sim 20 \%$ when different seed populations are used for the generation of the random numbers. For the solar maximum phase, the flux prediction efficiency of the model in the outer belt is comprised between +0.25 and -0.25 depending on the position and energy bin under consideration. This classifies the model as an average model (Turner et al. 2011).

In order to assess the consequences of the model definition to evaluated doses, the previously determined flux along the orbit was assumed to be impinging on a small silicon volume shielded by a finite aluminum slab of $0.5 \mathrm{~mm}$ thickness and the resulting electron dose was calculated. Figure 12a shows the electron dose absorbed in the silicon volume per half day as a function of time. The lower panel (Fig. 12b) shows the dose as it is accumulated in the silicon volume during the same period. For a three-year mission, the total absorbed dose in the silicon volume is predicted to be $16 \mathrm{krad}$ using the TOPmodel dynamic fluxes. The AE8-MAX model application estimated the total absorbed electron dose to $11 \mathrm{krad}$, using the SHIELDOSE-2 tool on the SPENVIS web site (http://www.spenvis.oma.be). The difference in dose is coming from the difference in the fluxes. 

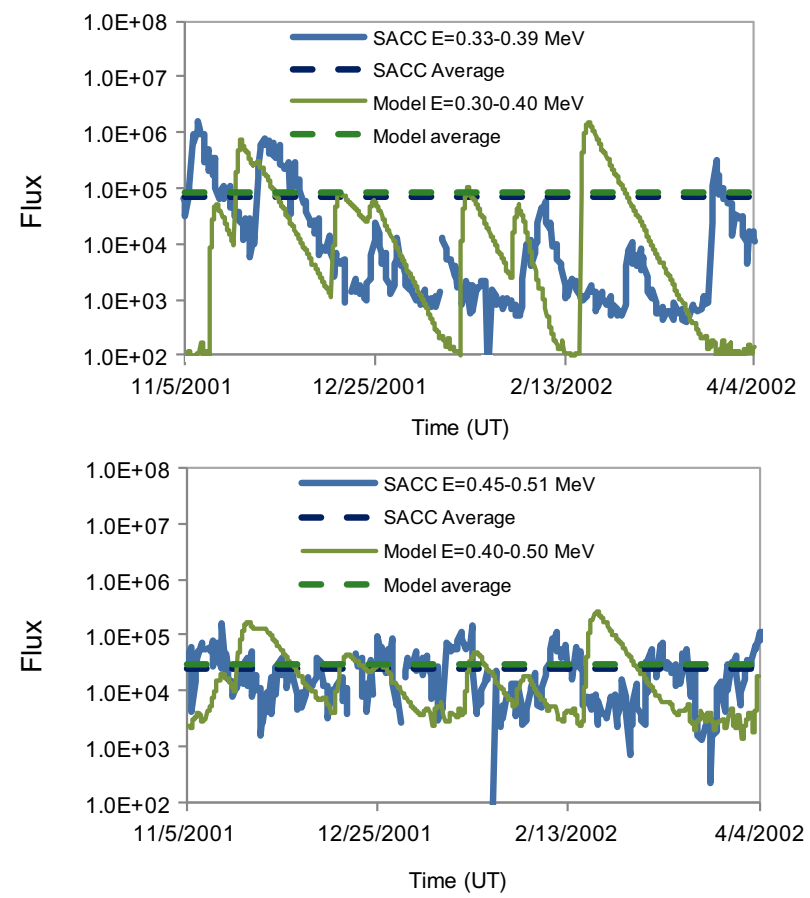

Fig. 11. The 0.5 day averaged fluxes (in $\mathrm{cm}^{-2} \mathrm{~s}^{-1} \mathrm{sr}^{-1} \mathrm{MeV}^{-1}$ ) as deduced from SAC-C data as a function of time for a period of 150 days (blue continuous lines) for two position bins $L=3.2-3.4$, $B=0.22-0.46 \mathrm{G}$ (upper panel) and $L=4.8-5.0, B=0.22-0.46 \mathrm{G}$ (lower panel). The discontinuous horizontal blue lines give the SAC$\mathrm{C}$ fluxes averaged over the 150 day time interval. The model results for 150 days of simulated data $(0.5$ day averaged date: green continuous line, 150 days averaged data: green discontinuous line) are superposed on the graph.

\section{Discussion}

The TOP-Model is not just a new way to predict in-orbit radiation belt particle fluxes; one advantage of this dynamic model over static models is that it mimics to a finer extent the actual behavior of the space radiation and this has consequences on radiation effects assessment as will be illustrated by two examples hereafter. The illustrative examples are based on the fluxes and doses evaluated along the above-defined case study orbit.

First example: Based on the particle fluxes shown in Figure 8 , it is possible to make an accurate statement on the number of times, within the 5 months time interval, a service will be interrupted provided that this service delivery requires that flux levels remain below a given critical level $\left(C_{\mathrm{L}}\right)$. Insurance companies may be interested in knowing that Earth Observation image acquisition may be interrupted twice at that position, if the filtering function on highly sensitive CCD-based camera cannot de-noise transients (Liebe 2001; Pickel et al. 2003) induced by a flux critical level $C_{\mathrm{L}}=10^{6}$ electrons/ $\left(\mathrm{cm}^{2} \mathrm{~s} \mathrm{MeV}\right)$ for the given energy range. The same service availability assessment may be performed based on the TOPmodel to determine Star Trackers performances in a LEO mission. Star Trackers exhibit difficulties in the processing of the images when exposed to enhanced radiation which may cause the appearance of "false stars" (Stauning et al. 2000). Unlike a static model, a dynamic model allows the determination of flux variability over a steady-state level and therefore the evaluation of the rate of space weather induced hazards and service interruption.

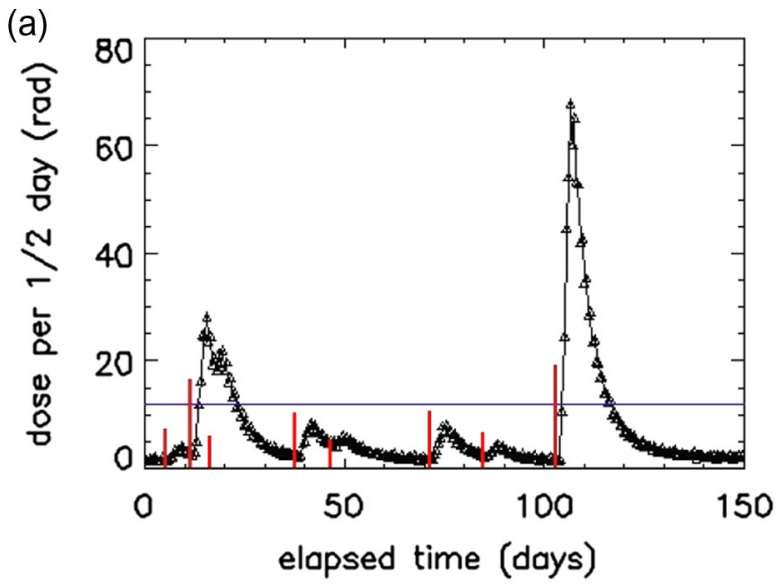

(b)

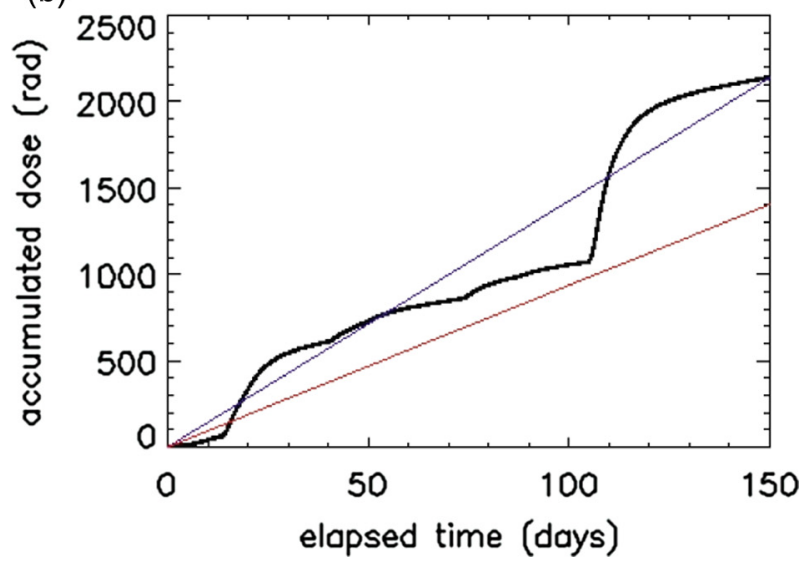

Fig. 12. The upper panel (a) shows the electron dose absorbed in the silicon volume per half day as a function of time. The blue line shows the averaged absorbed electron dose per half day. The thick red vertical lines indicate the time of occurrence of the geomagnetic storms. The lower panel (b) shows the dose as it is accumulated in the silicon volume during the irradiation period (black line). The blue line shows how this accumulated dose would evolve considering no dynamics. The red line shows the accumulated dose when using AE8.

Second example: From Figure 12a it can be observed that the dose rate is very variable over the 5 months time interval, switching from time of Low Dose Rate (LDR) to time of High Dose Rate (HDR). Following the detection of Enhanced Low Dose Rate Sensitivity (ELDRS), mainly in bipolar technology semiconductor devices, electronic system designers have suggested that Total Ionizing Dose (TID) evaluation tests of such sensitive components must be performed at LDR. In our case study, it can be observed that most of the time, the dose rate is low and therefore during the radiation hardness test campaigns of ELDRS sensitive devices, the results of the irradiation tests done at LDR would best approach the reality encountered in space on that orbit (Titus et al. 1999). However, this is not necessarily true for all mission orbits and a model which takes into account the variability of the encountered doses (such as the TOP-model) can best identify the appropriate dose rate scenario and give a more refined evaluation of dose effects on bipolar components.

More applications may be derived from the TOP-model: these include the evaluation of probability to accumulate a given dose after a given time period, probability to reach a given dose in one geomagnetic storm event (Fig. 12b), 
evaluation of the minimum flux/dose (steady-state conditions) along with an accurate forecasting of space weather. All of these are planned to be implemented in the final model in addition to proton fluxes.

\section{Conclusion}

The first version of a dynamic electron LEO radiation belt model has been developed. It is based on PDF of the storm occurrences, the flux variations, and their timescales. The model was found to be able to make a realistic prediction of the mean flux level encountered on orbit, and that it adds well the dynamic of the fluxes. It can predict the occurrence of steep dose enhancements per unit time as well as provide an estimation of the time fraction during which a device will be disabled due to exceeding fluxes (availability prediction). It also predicts that the dose rate is very variable within a given space mission and taking this into account may change the risk evaluation especially for devices that are ELDRS sensitive. Upon further development of the TOP-model, a more detailed comparison between model predictions and actual observations will be performed. Finally, an extension of the developed model will be elaborated to allow flux forecasting at a given position in space based on the real-time measured fluxes and the mapped particle lifetimes (after flux maximum has been reached and before the occurrence of a new storm event).

Presently the shortcomings of the model are the description of the flux level variations in the $(L<2.4, B>0.22 \mathrm{G})$ region, assumed to come from the resolution in the PDF of the flux variations. In an advanced version of the model, the data set will be extended to solve this issue. Furthermore, it will include a refinement in the position binning of the analyzed data, an extension of the covered regions (center of the SAA, high altitude fluxes) as well as the proton fluxes. So far, the only limitations for the further refinement of the TOP-model appear to be the availability of good data set.

Acknowledgements. The authors gratefully acknowledge the PRODEX team and Dr. W. Verschueren at BELSPO for supporting this research under Contract No. 90353. We also thank Drs. M. Parrot and J.-A. Sauvaud (CESR) for accepting us as guest investigator in the DEMETER mission of CNES. Drs. D. Boscher, $\mathrm{S}$. Bourdarie as well as R. Ecoffet are acknowledged for discussions and clarifications on the SAC-C/ICARE data. V. Pierrard thanks the European Commission's Seventh Framework Program (FP7/20072013) inside the grant agreement SWIFF (Project No. 2633430, http://www.swiff.eu).

\section{References}

Albert, J.M., N.P. Meredith, and R.B. Horne, Three-dimensional diffusion simulation of outer radiation belt electrons during the October 9, 1990, magnetic storm, J. Geophys. Res., 114, A09214, DOI: 10.1029/2009JA014336, 2009.

Benck, S., L. Mazzino, M. Cyamukungu, J. Cabrera, and V. Pierrard, Low altitude energetic electron lifetimes after enhanced magnetic activity as deduced from SAC-C and DEMETER data, Ann. Geophys., 28, 848-859, http://www.ann-geophys.net/28/849/ 2010/angeo-28-849-2010.html, 2010.

Beutier, T. and D. Boscher, A three dimensional analysis of the electron radiation belt by the Salammbô code, J. Geophys. Res., 100, 14853-14861, 1995.
Bourdarie, S., D. Boscher, D. Heynderickx, K. Stegen, P. Buehler, M. Cyamukungu, P. Stauning, S. Clucas, and R. Ecoffet, Radiation Environment Research From Multiple Monitors (RERMM), Final Report - Issue 1.0, ESA/ESTEC Contract No. 16709/02/NL/EC, p. 105-116, February, 2006.

Ecoffet, R., E. Lorfèvre, A. Corominas-murtra, A. Sicard-Piet, M. Moulin, et al., CNES Activities on Ionising Particle Measurements, Workshop on Ionising particle measurements in space, Noordwijk (The Netherlands), 31 January-2 February, 2005.

ECSS-E-ST-10-04C, Space engineering, Space environment, published by ESA-ESTEC Requirements and Standards Division, Noordwijk, The Netherlands, 15 November 2008.

Falguère, D., D. Boscher, T. Nuns, S. Duzellier, S. Bourdarie, R. Ecoffet, S. Barde, J. Cueto, C. Alonzo, and C. Hoffman, In-Flight observations of the radiation environment and its effects on devices in the SAC-C polar orbit, IEEE Trans. Nucl. Sci., 49 (6), 2782-2787, December 2002.

Fok, M.-C., R.B. Horne, N.P. Meredith, and S.A. Glauert, Radiation Belt Environment model: Application to space weather nowcasting, J. Geophys. Res., 113, A03S08, DOI: 10.1029/2007JA012558, 2008.

Fung, S.F., Recent development in the NASA trapped radiation models, Radiation Belts: Models and Standards. In: Geophysical Monograph, 97, Edited by J.F., Lemaire, D. Heynderickx, and D.N. Baker. The American Geophysical Union, Washington, DC USA, p. 79-91, ISBN: 0-87590-079-8, 1996.

Glauert, S.A., and R.B. Horne, Calculation of pitch angle and energy diffusion coefficients with the PADIE code, J. Geophys. Res., 110, A042046, DOI: 042010.041029/042004JA010851, 2005.

Kataoka, R., and Y. Miyoshi, Flux enhancement of radiation belt electrons during geomagnetic storms driven by coronal mass ejections and corotating interaction regions, Space Weather, 4, S09004, DOI: 10.1029/2005SW000211, 2006.

Koller, J., Y. Chen, G.D. Reeves, R.H.W. Friedel, T.E. Cayton, and J.A. Vrugt, Identifying the radiation belt source region by data assimilation, J. Geophys. Res., 112, A06244, DOI: 10.1029/2006JA012196, 2007.

Liebe, C. Ch., Charged particle-induced noise in camera systems, IEEE Trans. Nucl. Sci., 48 (4), p 1541-1549, 2001.

Ling, A.G., G.P. Ginet, R.V. Hilmer, and K.L. Perry, A neural network based geosynchronous relativistic electron flux forecasting model, Space Weather, 8, S09003, DOI: 10.1029/2010SW000576, 2010.

McIlwain, C.E., Processes Acting Upon Outer Zone Electrons, Radiation Belts: Models and Standards. In: Geophysical Monograph, 97, Edited by J.F., Lemaire, D. Heynderickx, and D.N. Baker. ISBN 0-87590-079-8, The American Geophysical Union, Washington, DC USA, p. 15-26, 1996.

O'Brien, T.P., and R.L. McPherron, An empirical dynamic equation for energetic electrons at geosynchronous orbit, J. Geophys. Res., 108 (A3), 1137, DOI: 10.1029/2002JA009324, 2003.

Parrot, M., Preface - Special issue of planetary and space science "DEMETER", Planet. Space Sci., 54, 411-412, 2006.

Pickel, J.C., A.H. Kalma, G.R. Hopkinson, and J. Ch. Marshall, Radiation effects on photonic imagers - A historical perspective, IEEE Trans. Nucl. Sci., 50 (3), p.671-688, 2003.

Sauvaud, J.A., T. Moreau, R. Maggiolo, et al., High-energy electron detection onboard DEMETER: The IDP spectrometer, description and first results on the inner belt, Planet. Space Sci., 54, 502-511, 2006.

Stauning, P., P. Davidsen, and M. Cyamukungu, High-Energy Particle Radiation Effects in the Instruments and Memory Circuits of Low-altitude Satellites, ESA Utilization Workshop, STEC, Noordwijk, 12 Dec 2000, http://web.dmi.dk/fsweb/ Esautilw/Radeff.html, 2000.

Subbotin, D.A., and Y.Y. Shprits, Three-dimensional modeling of the radiation belts using the Versatile Electron Radiation Belt (VERB) code, Space Weather, 7, S10001, DOI: 10.1029/2008SW000452, 2009 . 
Titus, J.L., D. Emily, J.F. Krieg, T. Turflinger, R.L. Pease, and A. Campbell, Enhanced Low Dose Rate Sensitivity (ELDRS) of linear circuits in a space environment, IEEE Trans. Nucl. Sci., 46 (6), p. 1608-1615, 1999.

Turner, D.L., X. Li, E. Burin des Roziers, and S. Monk, An improved forecast system for relativistic electrons at geosynchronous orbit, Space Weather, 9, S06003,

DOI: 10.1029/2010SW000647, 2011.

Vette, J.I., The AE-8 Trapped Electron Model Environment. NSSDC/ $W D C-A-R \& S$ 91-24, NASA/GSFC, Greenbelt, Maryland, November 1991.

Cite this article as: Benck S, Cyamukungu M, Cabrera J, Mazzino L \& Pierrard V: The transient observation-based particle (TOP) model and its potential application in radiation effects evaluation. J. Space Weather Space Clim., 2013, 3, A03. 